\title{
Predicting preadolescence alcohol use with data from a birth cohort study: association with socio-emotional-behavioral symptoms collected at age 7
}

João Picoito ${ }^{1,2}$, Constança Santos ${ }^{2,3}$, Carla Nunes ${ }^{2}$

1-Department of Child and Adolescent Psychiatry, Hospital Pediátrico de Coimbra, Portugal

2-Escola Nacional de Saúde Pública, Universidade NOVA de Lisboa, Portugal

3-Department of Pediatrics, Centro Hospitalar Universitário Cova da Beira, Portugal

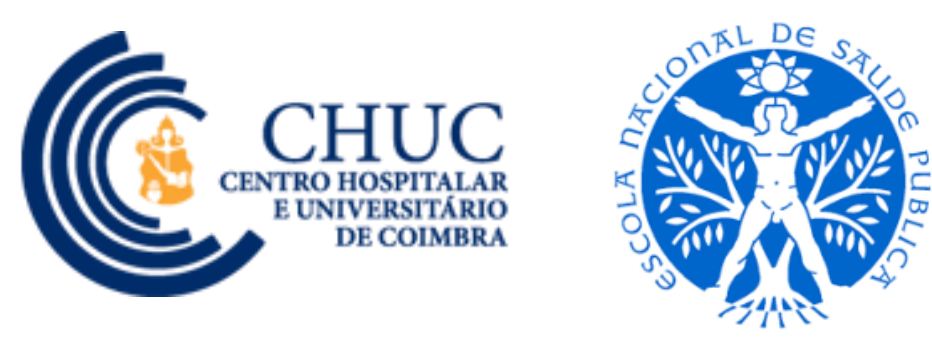

\section{Introduction}

Objectives: To predict preadolescence lifetime alcohol use. Identification of children at higher risk of risky behaviours in preadolescence and early adolescence is essential for effective prevention and health promotion strategies. Early initiation of substance use is associated with worse health outcomes and risky behaviours in adulthood.

\section{Methods}

Data for this study was drawn from the longitudinal study Millennium Cohort Study, held in the UK, and following a sample of children since their infancy. Logistic regression analyses stratified by gender, including 9703 children, were performed to predict preadolescence alcohol use (age 11) with predictors collected at age 7 , in an ecological framework.

\begin{abstract}
Results
Girls with more hyperactive symptoms (Odds ratio [OR] 1.09, [95\% confidence interval] 1.05 to 1.13 ) and peer problems (OR $1.05,1.09$ to 1.16) had higher odds of having used alcohol. Accordingly, boys who endorsed more hyperactive symptoms (OR 1.05, 1.02 to 1.09) had higher odds of ever drinking. In boys, but not in girls, harsh parenting was associated with drinking (OR 1.05, 1.02 to 1.08). Children living in monoparental families, displayed higher odds of alcohol drinking in preadolescence.
\end{abstract}

\section{Conclusions}

We found differences in the association of contextual and socioemotional-behavioral functioning with preadolescence alcohol use between boys and girls. Tailored interventions with an ecological framework are essential for effective prevention of early onset of alcohol use.

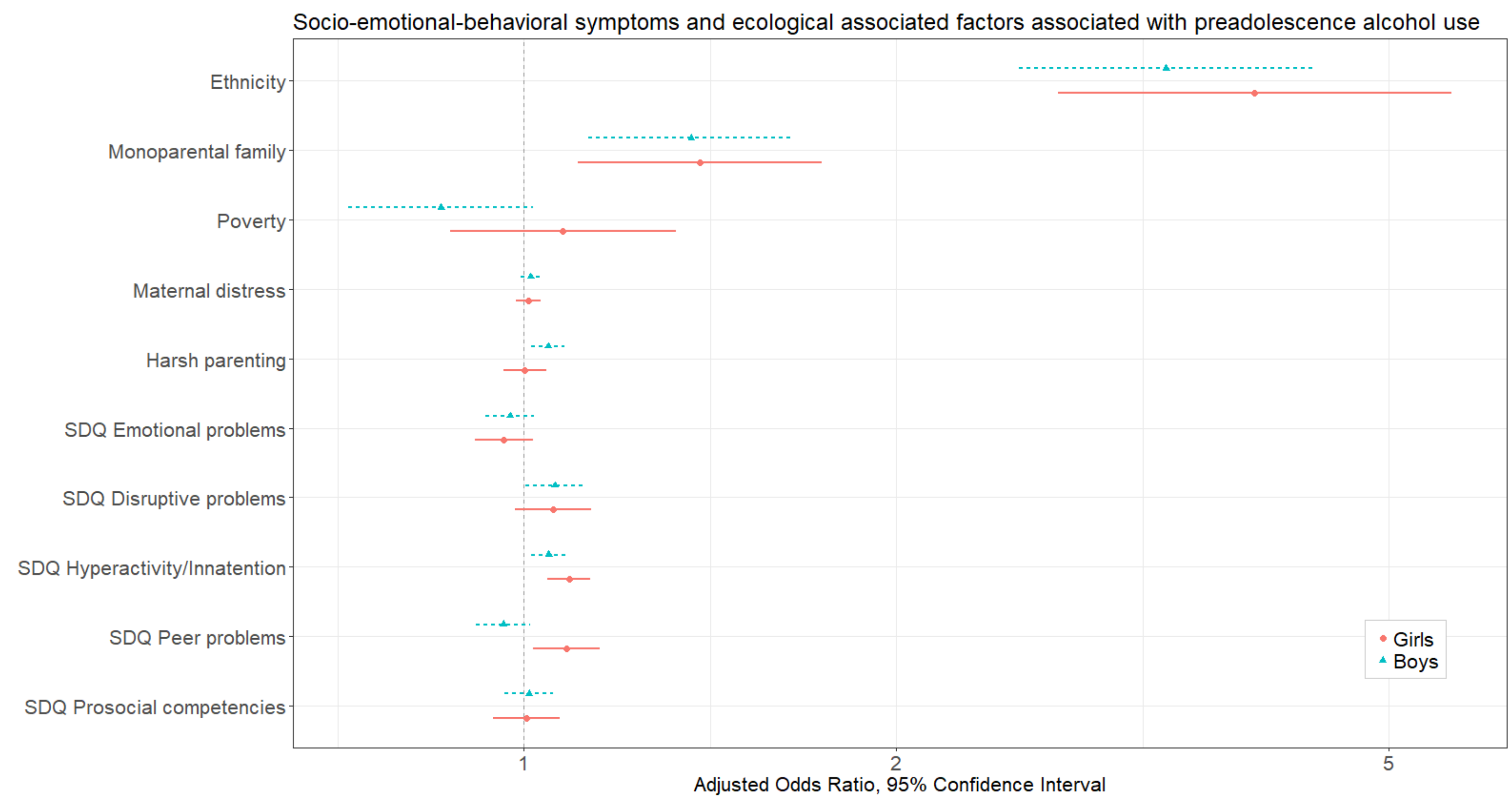

Legend: SDQ $=$ Strengths and Difficulties Questionnaire 